\title{
EDUCAÇÃO MATEMÁTICA: UM OLHAR HISTÓRICO-EPISTEMOLÓGICO
}

\author{
MATHEMATICAL EDUCATION: A HISTORICAL-EPISTEMOLOGICAL \\ LOOK
}

\author{
Francieli Cristina Agostinetto Antunes ${ }^{1}$ \\ Lisiane Cristina Amplatz ${ }^{2}$ \\ Maykon Jhonatan Schrenk ${ }^{3}$ \\ Sibeli Mallmann Pacheco ${ }^{4}$
}

\begin{abstract}
Resumo: Este texto é resultado de reflexões referentes ao tema "Educação Matemática como campo de pesquisa e profissional" em que, motivados por entender o que a compõe e como essas discussões foram se modificando ao longo do tempo, nos dedicamos a uma pesquisa bibliográfica não exaustiva para compreender o processo de construção deste campo de conhecimento, em âmbito nacional e internacional. Este estudo nos proporcionou conhecer como ocorreu a construção desta área e quais aspectos foram fundamentais em sua constituição como campo de pesquisa e profissional até os dias atuais. Além disso, ressaltamos a importância de manter vínculos entre educadores matemáticos e matemáticos, bem como entre pesquisadores e professores de Matemática, a fim de constituir uma área, profissional e acadêmica, cujos esforços estejam voltados para a produção de práticas que beneficiem o processo de ensino e aprendizagem da Matemática.
\end{abstract}

Palavras-chave: Educação Matemática; Campo Científico; Campo Profissional; Formação Docente.

\begin{abstract}
This text is the result of reflections regarding "Mathematics Education as a field of research and professional" in which, motivated to understand what makes it up and how these discussions have changed over time, we dedicate ourselves to a non-exhaustive bibliographical research to understand the process of construction of this field of knowledge at the international and national levels. This study allowed us to know how the construction of this area occurred and what aspects were fundamental in its constitution as a field of research and professional up to the present day. In addition, we emphasize the importance of maintaining the links between mathematical and mathematical educators, as well as between researchers and Mathematics teachers, in order to constitute an area, professional and academic, whose efforts are focused on the production of practices that benefit the process of teaching and learning mathematics.
\end{abstract}

Keywords: Mathematics Education; Scientific Field; Professional Field; Teacher Formation.

1 Doutoranda no Programa de Pós-Graduação em Educação em Ciências e Educação Matemática (PPGECEM), Universidade Estadual do Oeste do Paraná (UNIOESTE), Cascavel, PR, Brasil. E-mail: francieliantudes@gmail.com

2 Mestranda no Programa de Pós-Graduação em Educação em Ciências e Educação Matemática (PPGECEM), Universidade Estadual do Oeste do Paraná (UNIOESTE), Cascavel, PR, Brasil. E-mail: lisianeca@gmail.com

3 Mestrando no Programa de Pós-Graduação em Educação em Ciências e Educação Matemática (PPGECEM), Universidade Estadual do Oeste do Paraná (UNIOESTE), Cascavel, PR, Brasil. E-mail: maykon_schrenk@hotmail.com

4 Mestranda no Programa de Pós-Graduação em Educação em Ciências e Educação Matemática (PPGECEM), Universidade Estadual do Oeste do Paraná (UNIOESTE), Cascavel, PR, Brasil. E-mail: sibelimallmann@hotmail.com 


\section{Introdução}

Este texto é um conjunto de reflexões referentes ao tema "Educação Matemática como campo de pesquisa e profissional" em um ambiente de aprendizagem de um curso de pós-graduação, em que nos dedicamos a responder ao questionamento que nos foi apresentado: "O que compõe a Educação Matemática e como ocorreu historicamente essa construção?"

No intuito de responder ao questionamento que deu start a este texto buscamos compreender como ocorreu o processo de construção da Educação Matemática como campo de pesquisa e profissional, em âmbito nacional e internacional. Trilhamos uma investigação que nos remeteu a reflexões sobre percurso histórico do ensino de Matemática, no Brasil e fora dele, buscando identificar e entender como as discussões sobre o campo de conhecimento Educação Matemática foram se modificando ao longo do tempo, enquanto campo profissional e científico.

Neste sentido, nos dedicamos a uma pesquisa bibliográfica a partir dos apontamentos e questões em aberto presentes no texto de Miguel e colaboradores (MIGUEL et al., 2004), em busca de compreender o processo de construção da Educação Matemática, que segundo Fiorentini e Lorenzato (2006), embora sejam atribuídos a ela objetivos múltiplos, pode ser dividida em duas áreas gerais: a de natureza pragmática, que visa a melhoria da qualidade do ensino e da aprendizagem, e de natureza científica, que busca desenvolvimento da Educação Matemática como campo de investigação e de produção do conhecimento. Assim, no texto nos referiremos as áreas como campo profissional e de pesquisa, respectivamente.

Este estudo, cujas buscas foram realizadas nos periódicos disponíveis no Portal de Periódicos da Capes, nos proporcionou entender como ocorreu a construção da área Educação Matemática e quais aspectos foram fundamentais nesta constituição, tanto como campo profissional, quanto de pesquisa. Vários episódios foram relevantes nesse processo, desde os ocorridos na Idade Moderna, com maior influência das três revoluções desenroladas nesse período: Revolução Industrial (1767), a Revolução Americana (1776) e a Revolução Francesa (1789). A necessidade de novos processos de manufatura e de mão de obra especializada promovida na Revolução Industrial demonstrou a conveniência de maior número de instituições para promover o ensino, seguida por reflexões geradas pela necessidade, também, de melhorar o ensino já existente (MIGUEL et al., 2004). 


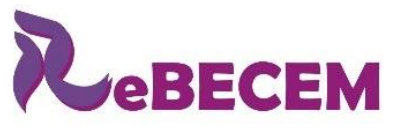

Revista Brasileira de Educação em

Ciências e Educação Matemática

DOI: http://dx.doi.org/10.33238/ReBECEM.2020.v.4.n.1.24069

O ensino passou por várias transformações ao longo da história, por meio de reflexões e contribuições de diversos pesquisadores e estudiosos da área. Alguns pesquisadores tiveram participação mais significativa nesse processo. Entre eles, podemos citar o filósofo americano John Dewey (1859-1952), que demonstrou preocupação com a necessidade de privilegiar a capacidade de pensar dos alunos, os quais deveriam ser preparados pelo professor para questionar a realidade. Relevante também foi a atitude do casal Grace C. Young e William H. Young ao publicar em 1904 um livro que propunha trabalhos manuais para auxiliar no ensino de geometria, escrito com intuito inicial de sanar as dificuldades na aprendizagem demonstrada pelos próprios filhos (MIGUEL et al., 2004).

Destaque para o matemático alemão Felix Klein (1849-1925), que defendeu que as escolas precisavam atentar mais às bases psicológicas que às sistemáticas no ensino de Matemática e, ainda, enfatizou que era competência do professor levar em conta o processo psíquico do aluno, apresentando os conceitos de uma maneira intuitivamente compreensível. No Brasil, influenciado pelas discussões promovidas por Felix Klein, Euclides Roxo (1890-1950) que durante sua vida acadêmica teve facilidade com a Matemática mas presenciou a dificuldade que grande parte dos seus colegas tinham em entende-la, na posição de professor de Matemática propõe uma reforma curricular e metodológica no ensino dessa Ciência. Citamos aqui alguns exemplos de pessoas dedicaram energia em reflexões e debatas no tangente a teoria e a prática, dos aspectos relacionados ao ensino e aprendizagem de Matemática.

Observação também relevante foi feita por Willian Higginson (1938-2008), quando apresentou sua observação da Educação Matemática a partir de um modelo, por meio da representação de um sólido geométrico, o Tetraedro, cujas faces representam as disciplinas, que, em seu entendimento, compunham a Educação Matemática. Chamou o Tetraedro de MAPS, sendo as faces denominadas como: M - Matemática, A - Filosofia, P - Psicologia, S - Sociologia. Porém, esse modelo foi superado por outros, representados por outras figuras geométricas, compostas por mais faces, pois, outras disciplinas foram sendo consideradas relevantes na formação do campo da Educação Matemática, tais como: Antropologia, História da Matemática, Psicologia e Didática.

O texto faz um passeio pela história da Educação Matemática, desde sua gênese, em âmbito nacional e internacional, observando sua influência no campo profissional e científico brasileiro, buscando observar e compreender as causas e as consequências da instituição do campo da Educação Matemática. 


\section{A Gênese}

Em busca de entender aspectos relacionados à Educação Matemática, sentimos a necessidade de compreender sua construção histórica, envolto a aspectos da matemática e da educação. Percebemos que a Educação Matemática, enquanto campo profissional, como afirma Kilpatrick (1996), “[...] é antiga. A Matemática tem sido ensinada desde que ela tem existência" (KILPATRICK, 1996, p. 111). Já como campo acadêmico, o autor relata que "[...] tem uma curta história que difere de país para país" (KILPATRICK, 1996, p. 100) e suas raízes “[...] tem menos do que um século" (KILPATRICK, 1996, p. 111).

Reflexões sobre o ensino de Matemática ocorreram ainda na Idade Média, mas se intensificaram em momentos que surgiram necessidades específicas, com reflexões iniciais no período da Idade Média e com o Renascimento na Idade Moderna. Na visão de Franco (1976), o Renascimento representou o rompimento com a Idade Média, uma vez que pregava o individualismo ao invés do coletivismo; o racionalismo ${ }^{5}$, ao invés do misticismo; o ecletismo ${ }^{6}$, ao invés da especialização; o mundanismo ${ }^{7}$, ao invés da reclusão, e opôs à religiosidade medieval ao humanismo ${ }^{8}$, exaltação do homem e sua obra. Aponta ainda que o homem renascentista, diferentemente do medieval, procurava viver com alegria, riqueza e aproveitava todos os prazeres que seu mundo oferecia.

Na Idade Moderna, intensificada com as três grandes revoluções: a Revolução Industrial (1767), a Revolução Americana (1776) e a Revolução Francesa (1789), que Miguel et al. (2004) atenta para a concepção da Educação Matemática como área prioritária em educação, ocorrida entre os séculos XIX e XX, com John Dewey, ao publicar, em 1895, em seu livro Psicologia do número, a ideia contra o formalismo, iniciando a cooperação entre aluno e professor, uma integração entre todas as disciplinas.

Os autores destacam, ainda, que na década de 1900 já se observava problemas na aprendizagem de conceitos matemáticos trabalhados na escola. Esses conflitos de opiniões sobre o ensino foram importantes para que conceituados pesquisadores matemáticos se interessassem por refletir sobre aspectos ligados ao ensino da Matemática,

5 Racionalismo que vê no pensamento, na razão, a fonte principal do conhecimento humano (HESSEN, 1980).

6 Vale-se de liberdade e pluralidade compositiva, não se atendo unicamente a essa ou aquela escola (LORENZONI, 2015).

7 Mundanismo, chega em nossa mente e em nosso coração de forma sorrateira, subtraindo a santidade que Deus deseja para pessoas e famílias (OXENDEN, 2017).

8 Humanismo, constituição de um sujeito em seu vínculo com o legado histórico de um mundo comum (CARVALHO, 2017). 
DOI: http://dx.doi.org/10.33238/ReBECEM.2020.v.4.n.1.24069

alguns talvez preocupados com a educação dos próprios filhos, como o caso do casal Young, anteriormente citado, e outros profissionais preocupados com a aprendizagem de Matemática da população em geral (MIGUEL et al., 2004).

A Matemática como campo profissional tem reconhecimento histórico desde a Idade Média, momento em que o olhar era ainda voltado às tarefas, procedimentos de sala de aula e construção de manuais, mas como campo científico é mais recente. Uma indicação disso se verifica na realização dos congressos em Matemática, que teve sua primeira edição realizada na Suíça, em 1887. Em 1908, no Congresso em Roma, foi registrada a socialização de preocupações de alguns congressistas referentes ao ensino e aprendizagem da Matemática. Um dos matemáticos que participou das discussões foi o alemão Felix Klein (1849 - 1925). Sua contribuição, segundo Miguel et al. (2004), foi o passo mais importante para o estabelecimento da Educação Matemática como disciplina, uma vez que "[...] Klein defende uma apresentação nas escolas que se atenha mais a bases psicológicas que sistemáticas. Diz que o professor deve, por assim dizer, ser um diplomata, levando em conta o processo psíquico do aluno, para poder agarrar seu interesse" (MIGUEL et al., 2004, p. 72).

Foi nesse congresso em Roma, sob a liderança de Felix Klein, que matemáticos e professores de Matemática compartilharam a preocupação com a qualidade na divulgação das ideias matemáticas às novas gerações, se tornando a Educação Matemática, ainda não nomeada dessa maneira, consolidada como uma subárea da Matemática. A partir disso, foram instauradas as primeiras tentativas de se conversar e pensar em um espaço adequado para reflexões relacionadas ao ensino e aprendizagem de Matemática. Com esse enfoque foi criado, em 1920, o National Council of Teachers of Mathematics (NCTM), de acordo com Miguel et al. (2004). Embora a pesquisa relacionada a aspectos de ensino e aprendizagem de Matemática estivesse crescendo em intensidade, havia pouco espaço para os debates dos professores de Matemática.

O período pós-guerra foi acompanhado, também, por discussões no âmbito da Educação Matemática, ainda antes do campo de pesquisa receber esse nome, com propostas a inovação e alteração de currículos, ocasionando, também nesse período, o aumento de pesquisas na área, demonstrando preocupações com o ensino de Matemática. Tais questões contribuíram para o surgimento da Educação Matemática. Fiorentini e Lorenzato (2006), tomando como referência um estudo desenvolvido por Kilpatrick (1996), destacam três fatores determinantes para o surgimento da Educação Matemática como um campo profissional e científico: 


\begin{abstract}
O primeiro é atribuído à preocupação dos próprios matemáticos e educadores matemáticos sobre a qualidade da divulgação/socialização das ideias matemáticas às novas gerações. Essa preocupação dizia respeito tanto à melhoria de suas aulas quanto à atualização/modernização do currículo escolar de matemática. [...] O segundo fato é atribuído à iniciativa das universidades europeias do final do século XIX, em promover institucionalmente a formação de professores secundários. Isso contribuiu para o surgimento de especialistas universitários em ensino de matemática. $\mathrm{O}$ terceiro fato diz respeito aos estudos experimentais realizados por psicólogos americanos e europeus, desde o início do século XX, sobre o modo como as crianças aprendiam a matemática (FIORENTINI; LORENZATO, 2006, p. 6).
\end{abstract}

O Movimento da Matemática Moderna trouxe discussões e reformulações ao currículo escolar, voltados para uma visão "internalista" da Matemática, em que o professor atuava como personagem central do ensino e privilegiava a demonstração dos conteúdos em sala de aula. Desta forma, este movimento buscava aproximar a Matemática da Escola Básica com a Matemática produzida pelos pesquisadores da áre, buscando suprir dificuldades com a aprendizagem de Matemática com mais Matemática.

O movimento foi criticado por muitos, entre eles o professor e historiados de Matemática Morris Kline (1908 -1992) e Wielewski (2008) que disse que foram inseridos “[...] no currículo conteúdos matemáticos que até aquela época não faziam parte do programa escolar como, por exemplo, estruturas algébricas, teoria dos conjuntos, topologia, transformações geométricas" (WIELEWSKI, 2008, p. 1). No entanto, esta proposta não solucionou os problemas do ensino.

Com a intensificação das críticas ao movimento, o grupo voltado à Educação Matemática fortaleceu suas discussões, enfatizando a necessidade de considerar outros aspectos envolvidos no processo de ensino e aprendizagem, como a capacidade cognitiva do sujeito que aprende, a sua cultura, os fatores sociais e econômicos, a língua materna e outros.

A preocupação e dedicação em se questionar sobre as relações e determinações entre ensino, aprendizagem e conhecimento matemático em um contexto específico, contribuiu para a união de profissionais, matemáticos e professores de Matemática, com preocupações convergentes, até que no ano de 1969, um marco para a Educação Matemática ocorreu na França, a realização do Primeiro Congresso Internacional de Educação Matemática (ICME). A década foi marcada pela criação da primeira revista de divulgação de pesquisas em Educação Matemática, Journal of Research in Mathematics Education (JRME), pois se entendeu que “a Educação Matemática não é apenas um campo profissional, mas também uma área de conhecimento, ou seja, a Educação 


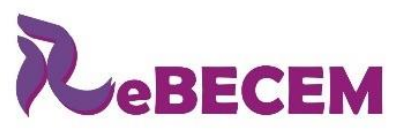

Revista Brasileira de Educação em

Ciências e Educação Matemática

DOI: http://dx.doi.org/10.33238/ReBECEM.2020.v.4.n.1.24069

Matemática é tanto uma área de pesquisa teórica quanto uma área de atuação prática, além de ser, ao mesmo tempo, ciência, arte e prática social" (FIORENTINI; LORENZATO, 2006, p. 14).

Embora há bastante tempo venha sendo discutido sobre os aspectos relacionados ao ensino e aprendizagem de Matemática, Kilpatrick (1996) destaca que do lado acadêmico, a questão do que é considerada pesquisa está ainda sendo debatida, do lado profissional, a Educação Matemática deve inevitavelmente se preocupar com a aplicação do conhecimento especializado para auxiliar os estudantes e os professores que são seus clientes. "A educação se tornou mais profissional, ela também se tornou mais científica, embora obviamente ela seja inevitavelmente uma ciência humana aplicada" (KILPATRICK, 1996, p. 113).

A concordância de que o ensino de Matemática precisava ser revisto trilhou, segundo Godoy (2015), um percurso histórico longo, marcado por diferentes concepções sobre seu ensino. O século XXI foi marcado pela concepção da Educação Matemática como campo científico, representando os esforços de compreender os fenômenos que ocorrem no interior da sala de aula, referente aos processos de ensino e aprendizagem da Matemática.

As discussões e avanços na área da Educação Matemática se estenderam em todo o globo, incluindo o Brasil, que teve mudanças significativas na formação dos profissionais e na implantação de um campo de pesquisa nacional, como veremos na próxima seção.

\section{Educação Matemática no Brasil}

No Brasil, segundo Fiorentini e Lorenzato (2006), a Educação Matemática passou por quatro fases: 1) constatação como campo profissional (período anterior à 1970); 2) nascimento (década de 1970 e início da década de 1980); 3) emergência de uma comunidade de educadores matemáticos (década de 1980); e 4) emergência de uma comunidade científica em Educação Matemática (anos de 1990).

Alguns movimentos influenciaram a construção do campo profissional, um deles foi o da "Escola Nova" que, a partir da década de 1920, deu início a criação dos primeiros manuais de orientação didático-pedagógica de Matemática. Os “educadores" matemáticos dessa época não focaram a pesquisa, mas a produção de livros-texto para os alunos e a escrita de orientações didático-metodológicas destinada aos professores. 


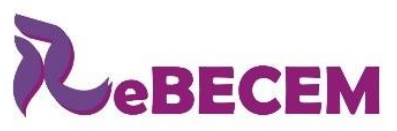

Revista Brasileira de Educação em

Ciências e Educação Matemática

DOI: http://dx.doi.org/10.33238/ReBECEM.2020.v.4.n.1.24069

Um nome importante desse período foi Euclides Roxo (1890-1950). "Euclides

Roxo, professor de matemática e diretor do Colégio Pedro II, foi o introdutor dos debates ocorridos nos congressos internacionais sobre a reforma do ensino de matemática" (VALENTE, 2005, p. 90).

O Movimento da Matemática Moderna influenciou a realização de cinco CBEMs - Congresso Brasileiro de Ensino de Matemática, entre 1955 e 1961, envolvendo matemáticos, professores de Matemática e pedagogos, com atenções voltadas ao ensino de Matemática no ensino primário e secundário. Porém, não chegou a sistematizar estudos envolvendo o ensino e aprendizagem de Matemática (FIORENTINI; LORENZATO, 2006).

Outros fatores contribuíram para o nascimento da Educação Matemática, como destacado por Fiorentini e Lorenzato (2006), são eles: o surgimento dos cursos de licenciatura em Matemática na década de 1920, criação de ginásios de aplicação na década de 1940, e a obrigatoriedade da disciplina de prática de ensino e estágio supervisionado na década de 1960, abrindo um campo profissional nas universidades para especialistas em didática e metodologia do ensino de Matemática.

A segunda fase, compreendida entre os anos iniciais da década de 1970 e os primeiros anos da década de 1980, entendida como surgimento da Educação Matemática como campo profissional, tendo a valorização da educação feita pelo regime militar, ampliação dos cursos de licenciatura e implantação de programas de pós-graduação em educação e psicologia. As pesquisas, segundo os autores, tinham ênfase pragmática e quantitativa e o ensino baseado no tecnicismo.

A terceira fase é marcada pela ampliação dos temas de estudo em Educação Matemática, a criação do primeiro curso de pós-graduação na área (Unesp - Rio Claro) e, com intuito de agregar uma comunidade de professores e educadores, em 1987 foi realizado o I Encontro Nacional de Educação Matemática (ENEM), depois a fundação da Sociedade Brasileira de Educação Matemática (SBEM), em 1988 (MIGUEL et al., 2004, p. 80). Porém, as pesquisas em Educação Matemática na década de 1980 se mostravam diversificadas, pulverizadas. Segundo Fiorentini e Lorenzato (2006), isso pode estar relacionado à falta de formação específica em Educação Matemática, sendo a grande maioria dos pesquisadores doutores em Matemática e psicologia.

Ainda segundo os autores, este período foi marcado pelo surgimento de linhas de pesquisa, tais como: etnomatemática, Modelagem Matemática, resolução de problemas, cognição Matemática relacionada aos contextos socioculturais, à prática pedagógica e a 


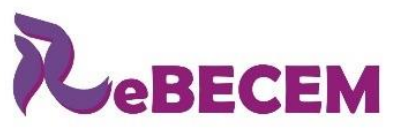

Revista Brasileira de Educação em

Ciências e Educação Matemática

DOI: http://dx.doi.org/10.33238/ReBECEM.2020.v.4.n.1.24069

formação de professores. Outras regiões de inquérito passaram a compor a Educação Matemática: a dimensão histórico-filosófica, a epistemologia, a antropologia sociologia, e a teleológico-axiológica. "Foi somente depois de todos esses acontecimentos que os educadores matemáticos passaram a identificar-se como tal e a interrogar sobre a natureza desse novo campo profissional" (FIORENTINI; LOREZATO, 2006, p. 32). Os questionamentos dos pesquisadores deixaram de estar pautados em "como ensinar" e passaram a ser "por que, para que e para quem ensinar?", priorizando aspectos mais amplos do âmbito educacional.

A quarta fase apresentada por Fiorentini e Lorenzato (2006) foi marcada pelo estabelecimento de uma comunidade científica, pois, no início dos anos 1990, retornaram ao Brasil mais de 20 doutores formados fora do país, que se juntaram a um número ainda maior de doutores formados no âmbito nacional e aos pesquisadores oriundos de outras áreas que migraram para a Educação Matemática, passando a contar com mais de 200 pesquisadores dedicados à área.

No ano de 1997, um grupo de professores da Pontifícia Universidade Católica de São Paulo (PUC - SP) propôs à Associação Nacional de Pós-Graduação e Pesquisa em Educação (ANPED) a criação de um Grupo de Trabalho (GT) em Educação Matemática para aumentar o campo de discussão, visibilidade e divulgação das pesquisas. Declaram, ainda, ser a Educação Matemática uma prática social que não está diferenciada das demais no interior do espaço acadêmico, nem juridicamente estabelecida como campo profissional autônomo, tampouco, portanto, reconhecida como campo disciplinar. Tendo o conhecimento do meio no qual está inserida, observamos que o maior foco de resistência ao projeto de sua disciplinarização estaria situado no interior do próprio meio acadêmico, ou, mais particularmente, entre matemáticos profissionais e os profissionais da educação.

\section{Educação Matemática: um enfoque contemporâneo}

Na busca de uma melhor compreensão e interpretação da Educação Matemática nos dias de hoje, voltamos brevemente para a apresentação de Higginson (1980), o qual destaca a existência de uma questão de incompreensão entre matemáticos e educadores matemáticos: “[...] parece ser o sentimento de alguns pesquisadores matemáticos que nada mais do que matemática realmente conta na educação matemática" (HIGGINSON, 1980, p. 4, tradução nossa). O autor aponta que a Educação Matemática é constituída por quatro dimensões: a Matemática, a Psicologia, a Filosofia e a Sociologia. Essa 
DOI: http://dx.doi.org/10.33238/ReBECEM.2020.v.4.n.1.24069

configuração é nominada de "Modelo do Tetraedro de Higginson" (MAPS), cujas faces são constituídas pelas quatro áreas: M - Matemática, A - Filosofia, P - Psicologia e S Sociologia.

Falar sobre este modelo implica em compreender o papel de cada uma destas disciplinas, a fim de possíveis reflexões sobre o campo da Educação Matemática. Para o autor, o professor deve compreender o máximo do sujeito que puder, reconhecendo sua capacidade e limitação. Assim, a Psicologia se preocupa com a forma na qual o indivíduo aprende Matemática. A Sociologia lida com a influência de grupos de indivíduos e suas criações sobre esta experiência, os quais exercem influência sobre o ensino e aprendizagem. Além disso, considerando que toda atividade intelectual é baseada em algum tipo de filosofia, esta disciplina lida com as preocupações que envolvem a epistemologia, ontologia, ética, estética, teleologia e axiologia, além dos problemas da verdade, certeza e consistência lógica.

Seguindo essa linha da compreensão da Matemática como uma visão ampla, em conjunto com as demais áreas, Bicudo (1993) coloca:

\begin{abstract}
[...] preocupações com o compreender a Matemática, com o fazer Matemática, com as interpretações elaboradas sobre os significados sociais, culturais e históricos da Matemática [...]. As pesquisas elaboradas no horizonte da região de inquérito da Educação Matemática trabalham em torno dessas preocupações, interrogando o compreender matemático, o fazer matemático, os significados sociais, culturais e históricos da Matemática. São, portanto, pesquisas que solicitam domínio compreensivo de um vasto horizonte de conhecimentos da Psicologia, da História, da Filosofia e, certamente da Matemática (BICUDO, 1993, p. 20-21).
\end{abstract}

Reconhecendo a importância de tratar a Matemática, o seu ensino e a aprendizagem em um contexto que interagem com diversas áreas, volta-se o interesse em analisar as relações encontradas entre as faces do Tetraedro de Higginson.

\footnotetext{
A Educação Matemática está na interseção de vários campos científicos (matemática, psicologia, pedagogia, sociologia, epistemologia, ciências cognitivas, semiótica, etc.) ela tem seus próprios problemas e questões de estudo, não podendo simplesmente ser vista como aplicação de partículas desses campos (FIORENTINI; LORENZATO, 2006, p. 10).
}

No modelo tetraédrico de Higginson, há áreas específicas existentes nas relações entre as suas faces, ou seja, a partir das interações entre a Matemática, a Filosofia, a Psicologia e a Sociologia, características da Educação Matemática podem ser destacadas. Por exemplo: a aresta PS, que representa a área comum entre a Psicologia e a Sociologia, é o campo reconhecido da psicologia social. Também, o vértice MAP, que representa a 
DOI: http://dx.doi.org/10.33238/ReBECEM.2020.v.4.n.1.24069

área comum entre a Matemática, a Filosofia e a Psicologia, destaca-se o trabalho de Piaget na epistemologia genética (HIGGINSON, 1980).

Segundo Burak e Klüber (2008), a Educação Matemática, ao longo do tempo, vem passando por ampliações, muitas delas fruto de um momento histórico que acaba por integrar outras áreas do conhecimento, como a Antropologia, a Língua Materna, a História da Matemática, a Epistemologia e outras. “A Educação Matemática está sendo desenvolvida e isso deu origem a um processo dinâmico; isto é, a Educação Matemática não pode ser vista como uma entidade estática" (RIUS, 1989a, p. 35, tradução nossa). Dessa maneira, surgem novas representações da Educação Matemática que podem promover novas interações "entre as áreas que as constituem, as quais por sua vez, agem e interagem em uma relação de reciprocidade" (BURAK; KLÜBER, 2008, p. 97).

Alinhada a essa ideia, a Educação Matemática vem promovendo progresso no processo do ensino e aprendizagem de Matemática, buscando interação entre as diversas áreas/disciplinas e, também, soluções/alternativas para amenizar problemas de longa data. Afirmar que temos problemas disciplinares e de aprendizagem sempre foi uma postura recorrente de educadores, algumas vezes se portando como uma verdade, mas, também, servindo como fuga e ponto de resistência à adoção de novas práticas que trabalhem o foco do problema.

Compreendendo a necessidade de nós, educadores, nos mantermos atualizados, buscando possíveis reformulações de áreas clássicas da pesquisa, precisamos estar abertos à legitimação de novas questões que venham a surgir, permitindo que, efetivamente, possamos, por meio da Educação Matemática, alterar o cenário do ensino da Matemática no país, buscando levar os resultados obtidos no campo da pesquisa para o campo profissional.

\section{Educação Matemática como Campo Profissional}

A Matemática é uma ciência antiga e bem definida, tendo com uma de suas bases a lógica. Porém, a Educação Matemática, como campo de pesquisa, é recente, cujas concepções e teorias ainda estão sendo configuradas.

Como campo profissional, a Educação Matemática deve se preocupar com a formação Matemática dos professores e a aplicação dos conhecimentos científicos para auxiliar os alunos no processo de aprendizagem. Na visão de Kilpatrick (1996), “A formação de professores continua sendo a função maior da Educação Matemática, 


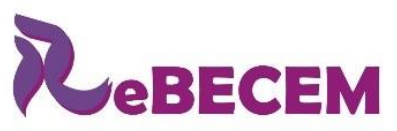

Revista Brasileira de Educação em

Ciências e Educação Matemática

DOI: http://dx.doi.org/10.33238/ReBECEM.2020.v.4.n.1.24069

paralelamente à busca do conhecimento sólido para ser aplicado" (KILPATRICK, 1996, p. 99). Em concordância com o autor, o período relevante para essa formação é tanto no âmbito da formação inicial quanto da formação continuada.

Para Kilpatrick (1996), uma profissão requer: 1) conhecimento especializado; 2) um caráter corporativo; 3) autodeterminação e autonomia; e 4) uma clientela. Em se tratando do ensino de Matemática, essas atribuições estão relacionadas ao conhecimento dos conceitos matemáticos e a relação entre eles; atribuir valor a organização escolar e trabalho colaborativo com os demais educadores; buscar diferentes metodologias e estratégias para o ensino de Matemática para seus alunos; a partir do conhecimento dos conceitos envolvidos e da busca de instrumentos e diferentes metodologias para o ensino é necessário ter a quem ensinar e que os sujeitos envolvidos no processo de ensino tenham a predisposição de aprender.

Como dito anteriormente, a Educação Matemática foi concebida inicialmente no campo profissional. Disciplinas voltadas à Educação Matemática foram estabelecidas nas universidades com o objetivo de contribuir na formação dos professores de Matemática, a partir disso que foi emergindo para o campo científico, especialmente com implantação de programas de pós-graduação na área.

A Educação Matemática como campo de pesquisa e como campo profissional não são disjuntas, Kilpatrick (1996) assegura que “[...] O lado científico não pode se desenvolver muito além, a menos que ele seja, de alguma forma, aplicado à prática profissional, e o desenvolvimento profissional requer o conhecimento especializado, que somente a investigação científica pode oferecer" (KILPATRICK, 1996, p. 112).

Ainda há dúvidas e confusões sobre a área de atuação do educador matemático, segundo Fiorentini e Lorenzato (2006), algumas vezes o professor de Matemática é chamado de matemático, mas nem sempre isso é verdade, pois suas funções têm origem epistemológica diferente. O matemático vê a Matemática com fim em si mesma, tem preocupações epistemológicas concernentes à Matemática e não ao ensino dela e, quando trabalha em cursos de formação, tende a fazê-lo deste lugar. O educador matemático visa promover uma educação pela Matemática, concebe a Matemática como meio para desenvolvimento intelectual e social do estudante, buscando contribuir para uma formação mais integral.

Na visão de Miguel et al. (2004), é competência da Educação Matemática pensar a formação de professores, estabelecendo parcerias para que ela não seja pensada isoladamente, mas em conjunto, um coletivo pensante. 


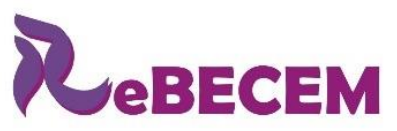

Revista Brasileira de Educação em

Ciências e Educação Matemática

DOI: http://dx.doi.org/10.33238/ReBECEM.2020.v.4.n.1.24069

Para o fortalecimento do campo da Educação Matemática, Kilpatrick (1996) traz sua opinião sobre a relevância de três aspectos: 1) que educadores matemáticos e matemáticos estabeleçam laços fortes de trabalho, em um clima de confiança e respeito; 2) que educadores matemáticos e professores de Matemática na prática em diferentes níveis de ensino também estabeleçam laços sólidos; e 3) tem maior desenvolvimento quando se torna um departamento distinto, exclusivo, em uma universidade.

\section{Algumas Considerações}

Consideramos, finalmente, que a Educação Matemática, enquanto campo de pesquisa e profissional, possa exercer efetiva contribuição na melhoria da qualidade dos processos de ensino e aprendizagem da Matemática. Concordamos com Burak e Klüber (2008) quando ressaltam que não é relevante padronizar a Educação Matemática, pois esta pluralidade das disciplinas que a constitui " [...] pode contribuir para as discussões e o avanço sobre a natureza da Educação Matemática" (BURAK; KLÜBER, 2008, p. 105).

Além disso, para que haja fortalecimento e crescimento deste campo, Kilpatrick (1996) ressalta a importância da manutenção dos laços entre educadores matemáticos e matemáticos, bem como entre pesquisadores e professores de Matemática, a fim de constituir uma área, profissional e acadêmica, cujos esforços estejam voltados para a produção de práticas que beneficiem o processo de ensino e aprendizagem da Matemática. Processo que vem crescendo no país pelo aumento na oferta de cursos de pós-graduação na área e a procura dos professores de Matemática por estes cursos, do qual também fazemos parte e esperamos contribuir para o fortalecimento, tanto como campo de pesquisa como campo profissional.

\section{Referências}

BICUDO, M. A. V. Pesquisa em Educação Matemática. Pro-posições, v. 4, n. 1, p. 18-23. São Paulo, 1993.

BURAK, D.; KLÜBER, T. E. Educação Matemática: contribuições para a compreensão da sua natureza. Acta Scientiae, Canoas, v. 10. n. 2, p. 93-106, jul./dez. 2008.

CARVALHO, J. S. F. de. Os ideais da formação humanista e o sentido da experiência escolar. Educ. Pesqui., São Paulo, v. 43, n. 4, p. 1023-1034, out./dez., 2017.

FIORENTINI, D.; LORENZATO, S. Investigação em Educação Matemática: percursos teóricos e metodológicos. 1. ed. Campinas: Autores Associados, 2006.

FRANCO, H. Jr. História das Civilizações. 2. ed. São Paulo: Atlas, 1976. 
GODOY, K. V. O papel e contribuições matemáticas das sociedades científicas do norte da Inglaterra em fins do século XVIII. In: ENCONTRO BRASILEIRO DE ESTUDANTES DE PÓS-GRADUAÇÃO EM EDUCAÇÃO MATEMÁTICA, 19., 2015. Anais... Juiz de Fora. Disponível em: http://www.ufff.br/ebrapem2015/files/2015/10/gd5_Kleyton_Godoy.pdf. Acesso em 05 jan. 2019.

HESSEN, J. A Origem do Conhecimento. In: HESSEN, Prof. Johannes. A Origem do Conhecimento. 7. ed. Coimbra/ Portugual; Armênio Amado, 1980. p. 58-85.

HIGGINSON, W. On The foundations of Mathematics Education. FLM Publishing Association Montreal Quebec Canada. For the learning of Mathematic. 1980.

KILPATRICK, J. Fincando Estacas: Uma tentativa de demarcar a Educação Matemática como campo profissional e científico. Tradução: Rosana G. S. Miskulin; Cármen Lúcia B. Passos; Regina C. Grando e Elisabeth A Araújo. Revisão Dario Fiorentini. Zetetiké, Campinas, v. 4, n. 5, p. 99-120, jan./jun. 1996.

LORENZONI, H. O. O Eclético. XI Semana de Extensão, Pesquisa e Pós-Graduação SEPesq, São Paulo. 2015.

MIGUEL, A.; D’AMBROSIO, U; GARNICA, A.; IGLIORI, S. B. C. A. Educação matemática: breve histórico, ações implementadas e questões sobre sua disciplinarização. Revista Brasileira de Educação, São Paulo, v. 27, p. 70-210. 2004.

OXENDEN, Ashton-1862-1902. Mundanismo. Ashton Oxenden Tradução, adaptação e edição por Silvio Dutra - Rio de Janeiro, 2017.

RIUS, E. B. La Educación matemática: Uma reflexión sobre su naturaleza y sobre su metologia. Educación Matemática. v. 1, n. 2, ago. 1989.

VALENTE, W. R. Euclides Roxo e história da educação matemática no Brasil. UNIÓN Revista Iberoamericana de Educacion Matemática. n. 1. p. 89-94, mar. 2005.

WIELEWSKI, G. D. O Movimento da Matemática Moderna e a Formação de Grupos de Professores de Matemática no Brasil. 2008. Disponível em:

http://www.apm.pt/files/_Co_Wielewski_4867d3f1d955d.pdf. Acesso em: 03 fev. 2020. p. 110.

Recebido em: 17 de fevereiro de 2020

Aceito em: 16 de março de 2020 